\title{
Is It Feasible To Apply Clinical Governance Principles In Operational Primary Care?
}

\author{
AJ Leach
}

\section{For Debate: the Application of Clinical Governance Principles in Operational Primary Care.} UK Civilian and military peacetime medical care is increasingly addressing the requirement for clinical governance. This has been defined as

"A framework through which organisations are accountable for continuously improving the quality of their services and safeguarding high standards of care by creating an environment in which excellence in clinical care will flourish"(1).

It is supported by the four pillars of clinical effectiveness, prof-essional development, continuous quality improvement and risk management. Individuals and systems in both the NHS and military medical services are currently working towards the goals of Clinical Governance to improve patient care. The aim of this paper is to discuss clinical governance in operational military primary care.

The core business of the Defence Medical Services is to support military operations. Military operations vary in their scope, risk and location and thus the range of medical skills required to support them can potentially be very different. Given these variables, is it feasible to implement Clinical Governance on operations?

Whilst the overarching term of Clinical Governance is relatively new, many of its concepts are not. The training of personnel for their military medical task is not new, nor is the assessment of risk (both medical and operational). For example an operational medical assessment will in effect state the standard of care to be given, the systems to be used to deliver the care, the potential risks and options to reduce the risks, if possible. What has changed is the explicit requirement to meet peacetime civilian medical standards, if at all possible during operational deployments. This requirement is easier to meet in some theatres but extremely difficult in others. Specific difficulties that are not routinely encountered in civilian practice are the requirements for some medical facilities to move, often at short notice and having to support very small isolated detachments with their own integrated medical support.

\section{So how are we doing?}

Clinical effectiveness requires practice to be based on evidence or best practice. It may be implemented through protocols and guide- lines. In some aspects the Defence Medical Services are doing very well (such as Casualty Treatment Regimens or the application of Battlefield Advanced Trauma Life Support), but in others, particularly in operational general practice, considerable work is still required. This applies to all members of staff, whether they are doctors, nurses or medics. Access to information is a key enabler. Both paper-based information (because it is simple and can be taken into virtually any location) and access to electronic information (because it is more likely to be up to date) are required to support clinical decision making. To date emphasis has been on the former, with limited application of the latter. To complicate matters, evidence based practice may not be implemented for a number of additional reasons; these include the personal views of the doctor, the relationship between the doctor and patient, and the interpretation of the evidence in the light of potential logistical difficulties, such as accessing secondary care (2). Thus simply giving information to the health professional may be insufficient. It is not just a case of asking people to use protocols; support and education are needed, if they are to be used when seeing patients.

\section{Professional Development}

The key elements are the identification of learning needs and addressing these through personal development plans, appraisal and assessment. This is an area that requires considerable work. Firstly there is the problem of having personnel adequately trained for task (which can be myriad in nature), but then providing the necessary supervision and assessment by appropriately trained individuals both before and during operations. As part of the Post Graduate Medical Officers Course considerable effort is made to prepare new medical officers for their first operational deployment. However, educational input primarily gives knowledge and whilst this is essential, the application of knowledge in a clinical environment is a much more complex and potentially fraught task. Efforts have been made to include clinical senior doctors, preferably with educational and supervisory skills, within the establishments of some operational theatres. They do not have a direct clinical responsibility for patients, but are able to oversee and advise. The deployment of "Senior Medical 
Officers" has been a success (DW Smith, personal communication). They provide support to medical officers and other primary care staff and are able to advise on the management of clinical, administrative and operational matters. However, communication difficulties and the distances between locations often limit supervision.

\section{Continuous Quality Improvement}

Continuous quality improvement seeks to ensure practice is kept in line with current guidelines and evidence. This is achieved by audit against agreed criteria, customer feedback leading to clear, documented action plans and follow-up to complete the audit cycle. Notwithstanding operational necessities, audit is possible on operational tours, but it is not as easy as in a peacetime or barracks location. Problems normally include a very mobile population, frequently a lack of accurate age/sex and morbidity registers and difficulties with patient records. Patient record difficulties have reduced since the implementation of the computer system "deployable EMIS", but in some operational circumstances it may not either be possible or appropriate to use the computer system to its full capacity. However, audits can frequently be undertaken into the "process of care" - 'was the right medication prescribed', or 'were the notes complete enough to allow another person to understand the consultation', as examples?

\section{Risk Management}

Risk management is the pro-active systematic approach that examines the processes of an organisation. It must identify and assess the level of risk by systematic analysis and significant event monitoring. Risks can then be eliminated or reduced through effective management, education and support. Of the four pillars, my personal view is that this is the area that requires the most work, albeit that it links with the other three pillars. Every healthcare system takes risks (whether it is aware of them or not) however, on operations, risks (both military and medical) often have to be taken in order to meet the overall aim of the mission. Active risk reduction can be facilitated by the use of Significant Event Analysis (SEA) and by having explicit risk assessments, both of which are possible on operational tours. For example in Bosnia during the summer of 2001 SEA were undertaken on the management of patients with acute asthma and on aspects of dispensing.

In terms of medical and related personnel, the major risk is that many are not appropriately trained for their role. In particular some medical officers are providing primary care services with little or no continuation training, and whose qualifications in primary care do not meet the requirements to practise in general practice (3). An argument frequently articulated is that military primary care is occupational medicine and thus doctors do not need to be qualified in general practice. Whilst some problems are occupational health related, many patients present with clinical difficulties that are not work related; for example psychiatric or situational difficulties, or contraception. These conditions could not be described as occupational medicine. MoD policy is enshrined within the Armed Forces Overarching Personnel Strategy:

"To provide Service personnel in the UK and overseas (and dependants accompanying Service personnel overseas) both in operational and nonoperational areas, with timely, appropriate access to healthcare of a clinical standard as close as possible to that pertaining in the National Health service, thereby facilitating their speedy return to duty” (4).

Thus if this standard of care is to be provided, primary care doctors should be trained general practitioners or if not, should have explicit supervision and support. Within NATO, there is a range of training given to medical officers. For example Canadian doctors are expected to be Board Certified Family Physicians (equivalent of general practitioners) before they are allowed to undertake a Regimental Medical Officer post. In contrast Dutch medical officers undertake training similar to the Post Graduate Medical Officer Course, however they are provided with supervision and have continued medical training during their general duties tour (I Zielmann, personal communication).

\section{So where does this leave us?}

Sir Michael Rawlins, the Chairman of NICE stated in 1998 that "It is implicit that Clinical Governance and the commitment it demands can never be wholly satisfied. As we get close to Utopia, the goal posts will change and more will be required of us". Clinical Governance can be used on operations, but it is purely a tool to ensure that patients are treated correctly, by appropriately trained personnel and that lessons are learnt from any mishaps, to ensure that they do not occur again. There are differences in the risk analysis from peacetime requirements as under some circumstances greater risks are allowable, however considerable work is required to ensure that improved systems are in place and that personnel are better trained for their task. In particular given the considerable shortfall in the number of medical personnel available, it may never be possible for all primary care doctors to be trained general practitioners, but this should be the aim.

Williams in his editorial in the Corps Journal (5) outlined the "revolution" occurring within the Army Medical Services. Clinical Governance is a journey, a journey that has no obvious destination but the 
direction of travel is clear. It is, however, a journey that we must travel. We owe it to our patients, both current and future, to ensure that this revolution is carried through.

The views and opinions expressed in this article are those of the author and imply no relationship to the MoD or AMS policy either present or future.

\section{References}

1. A First Class Service - Quality in the NHS. Department of Health 1998

2. Freeman AC, Sweeney K. Why general practitioners do not implement evidence: qualitative study. $B M \mathcal{F}$ 2001; 323: 1000-2.

3. The National Health Service (Vocational Training for General Medical Practice) Regulations 1997.

4. Armed Forces Overarching Personnel Strategy. Chapter 3 Annex B - Personnel Strategy Guidelines 19. Ministry of Defence July 1998. (http://www. mod.uk/issues/afops/chapter3annexb.htm)

5. Williams AN. The AMS revolution - primary care, quality assurance, capabilities and competence $\mathcal{F} R$ Army Med Corps 2001; 147. 3: 257-259. 